\title{
Rational design of soft mechanical metamaterials: Independent tailoring of elastic properties with randomness
}

M. J. Mirzaali, R. Hedayati, P. Vena, L. Vergani, M. Strano, and A. A. Zadpoor

Citation: Appl. Phys. Lett. 111, 051903 (2017); doi: 10.1063/1.4989441

View online: http://dx.doi.org/10.1063/1.4989441

View Table of Contents: http://aip.scitation.org/toc/apl/111/5

Published by the American Institute of Physics

\section{Articles you may be interested in}

A three-dimensional all-metal terahertz metamaterial perfect absorber

Applied Physics Letters 111, 051101 (2017); 10.1063/1.4996897

Broadband acoustic energy confinement in hierarchical sonic crystals composed of rotated square inclusions Applied Physics Letters 111, 054103 (2017); 10.1063/1.4985230

Wavelength-selective and diffuse infrared thermal emission mediated by magnetic polaritons from silicon carbide metasurfaces

Applied Physics Letters 111, 051904 (2017); 10.1063/1.4996865

Additively manufactured metallic pentamode meta-materials

Applied Physics Letters 110, 091905 (2017); 10.1063/1.4977561

Experimental validation of an ultra-thin metasurface cloak for hiding a metallic obstacle from an antenna radiation at low frequencies

Applied Physics Letters 111, 054105 (2017); 10.1063/1.4996065

Active tuning of high- $Q$ dielectric metasurfaces

Applied Physics Letters 111, 053102 (2017); 10.1063/1.4997301

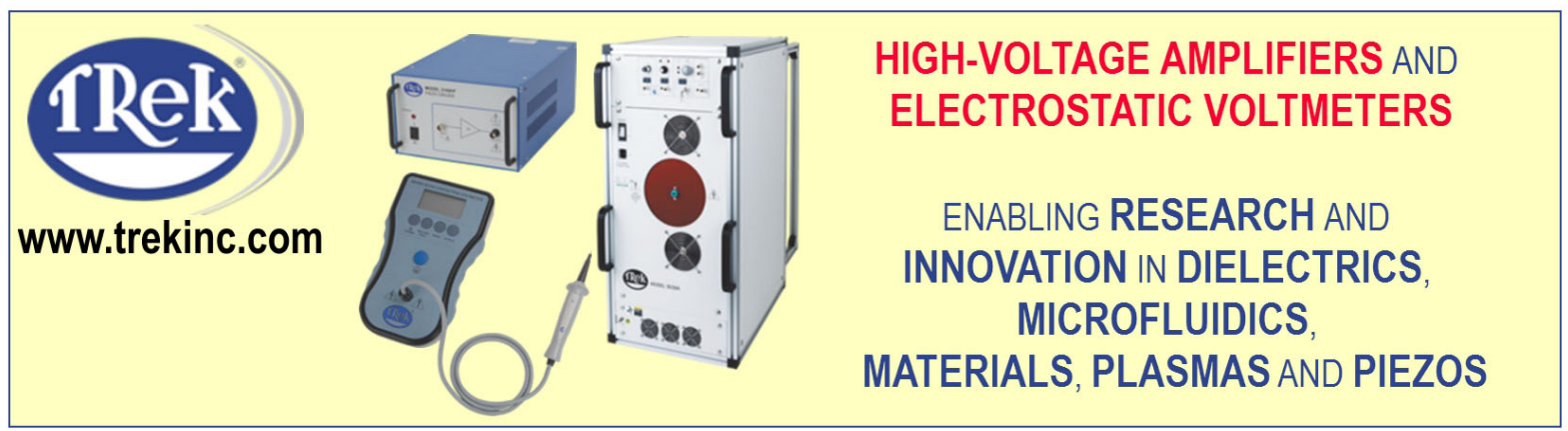




\title{
Rational design of soft mechanical metamaterials: Independent tailoring of elastic properties with randomness
}

\author{
M. J. Mirzaali, ${ }^{1,2, a), b)}$ R. Hedayati, ${ }^{2, b)}$ P. Vena, ${ }^{3}$ L. Vergani, ${ }^{1}$ M. Strano, ${ }^{1}$ and A. A. Zadpoor ${ }^{2}$ \\ ${ }^{1}$ Department of Mechanical Engineering, Politecnico di Milano, Via La Masa 1, 20156 Milano, Italy \\ ${ }^{2}$ Department of Biomechanical Engineering, Faculty of Mechanical, Maritime, and Materials Engineering, \\ Delft University of Technology (TU Delft), Mekelweg 2, 2628 CD Delft, The Netherlands \\ ${ }^{3}$ Department of Chemistry, Materials and Chemical Engineering Giulio Natta, Politecnico di Milano, \\ Piazza Leonardo da Vinci, 32, 20133 Milano, Italy
}

(Received 9 June 2017; accepted 23 July 2017; published online 4 August 2017)

\begin{abstract}
The elastic properties of mechanical metamaterials are direct functions of their topological designs. Rational design approaches based on computational models could, therefore, be used to devise topological designs that result in the desired properties. It is of particular importance to independently tailor the elastic modulus and Poisson's ratio of metamaterials. Here, we present patterned randomness as a strategy for independent tailoring of both properties. Soft mechanical metamaterials incorporating various types of patterned randomness were fabricated using an indirect additive manufacturing technique and mechanically tested. Computational models were also developed to predict the topology-property relationship in a wide range of proposed topologies. The results of this study show that patterned randomness allows for independent tailoring of the elastic properties and covering a broad area of the elastic modulus-Poisson's ratio plane. The uniform and homogenous topologies constitute the boundaries of the covered area, while topological designs with patterned randomness fill the enclosed area. Published by AIP Publishing. [http://dx.doi.org/10.1063/1.4989441]
\end{abstract}

Mechanical metamaterials ${ }^{1-4}$ are materials for which small-scale topological design determines the large-scale properties. It is, therefore, possible to adjust the topological design of such materials to obtain the desired set of mechanical properties including some unusual properties such as negative Poisson's ratio, ${ }^{5}$ negative compressibility, ${ }^{4}$ ultrahigh stiffness, ${ }^{6}$ and fluid-like behavior. ${ }^{7}$ Rational design is the process of applying physical principles, analytical solutions, and computational models to devise the topological design that gives rise to the desired set of mechanical properties. ${ }^{8-11}$

As far as the elastic properties of isotropic metamaterials are concerned, different topological designs result in different values of the elastic modulus and Poisson's ratio. It is, of course, possible to consider other duos of the elastic properties such as the elastic modulus and shear modulus or the bulk modulus. We will, however, use the elastic modulus and Poisson's ratio here due to their practical importance. While the elastic modulus is often used to evaluate the loadbearing capacity of the material, Poisson's ratio in general and auxetic metamaterials in particular ${ }^{12-16}$ are instrumental in designing mechanical metamaterials with advanced functionalities. ${ }^{15,17}$

Many applications of mechanical metamaterials require simultaneous adjustment of both the elastic modulus and Poisson's ratio, which is possible through the recent developments in additive manufacturing technologies in general and dual-material additive manufacturing in particular. ${ }^{18}$ However, topological designs that result in favorable values

\footnotetext{
${ }^{\text {a) }}$ Author to whom correspondence should be addressed: mirzaalimazandarani. mohammad@polimi.it and mirzaali.mohammad@gmail.com. Tel.: +390223998203. Fax: +392 23998263.

b) Both authors share first authorship.
}

of one property may adversely affect the values of the other. For example, it has been shown that topological designs that decrease Poisson's ratio to large negative values that are desired for many applications result in decreased values of the elastic modulus. ${ }^{15,17}$ It is therefore important to find rational design approaches through which the elastic modulus and Poisson's ratio could be independently adjusted within a wide range of values.

In this study, we propose an approach based on the random distribution of auxetic (re-entrant hexagonal honeycomb, A-UC) and conventional (periodic hexagonal, C-UC) unit cells in soft cellular metamaterials to cover particular areas in the elastic modulus-Poisson's ratio plane that cannot be covered with a simple arrangement of the same unit cells. The main hypothesis, therefore, is that "patterned randomness could be used as a rational design tool to achieve a wide range of elastic properties while keeping the unit cell design constant." We also propose an inexpensive method based on hobbyist 3D printers for indirect additive manufacturing of the proposed soft metamaterials. As it is generally not possible to print highly elastomeric materials with hobbyist 3D printers, the proposed manufacturing approach could facilitate the research and practical applications of soft mechanical metamaterials.

The dimensions of the auxetic (A-UC) and conventional (C-UC) unit cells are presented in the supplementary material. In the development of these unit cells, we assumed that the total length, 1 , and width, w, of both unit cells are constant. The only parameter that could change the geometry of both unit cells was the interior angle $(\theta)$ of each unit cell (see Fig. 1). Due to geometrical constraints (Table S1, supplementary material), the angle of unit cells could vary between $55^{\circ}$ and $130^{\circ}$. 
The final lattice structure contained a random combination of hexagonal and re-entrant unit cells. We propose two main algorithms to introduce randomness into the lattice structure, which is detailed in Table S2 (supplementary material) and Fig. 1. In Table S2 (supplementary material), $i$ and $j$ represent the column and row numbers, respectively, while $m$ and $n$ stand for the total number of columns and rows, i.e., $n w=W$ and $m l=L$. For the first group, we considered a uniform distribution of randomness. For the specimens in this group, we selected three levels of randomness as $25 \%, 50 \%$, and $75 \%$. These values show the probability that a unit cell in a fully A-UC lattice structure is replaced by a C-UC. In the second group, we introduced randomness in the structure using linear or radial patterns. A schematic illustration of each model is presented in Fig. S1 in the supplementary material.

Numerical simulation and modeling were performed with ANSYS ${ }^{\circledR}$ Academic Research, Release 16.2, software. A hyperelastic (Mooney-Rivlin) material model $\left(E_{s}\right.$ $=0.721 \mathrm{MPa}, \quad \nu_{s}=0.49, C_{10}=103927.05 \mathrm{~Pa}, \quad$ and $C_{01}$ $=-2591.52 \mathrm{~Pa})$ was used for simulations. The coefficients of the material model were determined based on the standard experimental protocols for testing elastomeric materials in tension (ASTM D412 Type C) and compression (ASTM D 575-91, Standard Test Methods for Rubber Properties in Compression). Timoshenko beam elements with rectangular cross-sections and constant thickness $(t=0.9 \mathrm{~mm})$ were employed for discretizing the geometries of the finite element (FE) models. The out-of-plane thickness of the lattice structure $\left(t_{\text {lattice }}\right)$ was set to $13 \mathrm{~mm}$.

Similar boundary conditions were applied to all FE models. Consistent with the experimental configurations and mechanical testing setup, the nodes located in the first row on the left side of the structure were fixed in all directions. A constant displacement in the $\mathrm{x}$-direction was applied on the right side of the geometry. The nodes located on the right side of the structure were constrained in both $\mathrm{Y}$ and $\mathrm{Z}$ directions. A non-linear solver was used for the simulations.

For the specimens with the uniform distribution of randomness, a random number was generated in the range of $\left(-1+\frac{P R}{100}, \frac{P R}{100}\right)$, where $P R$ stands for the percentage of randomness. The generated number shows the incident (a)

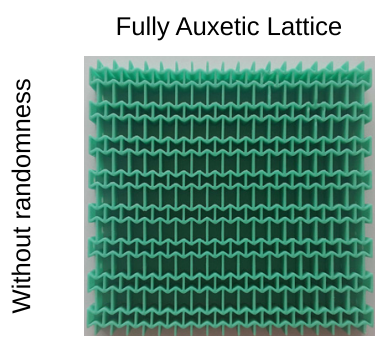

(b)

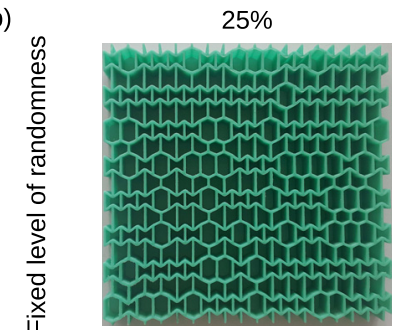

(c)

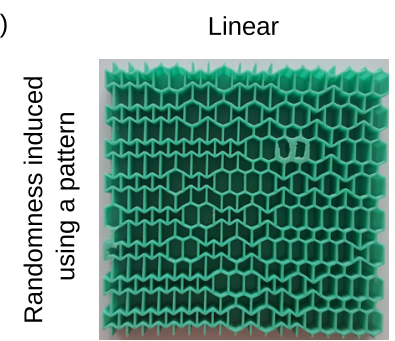

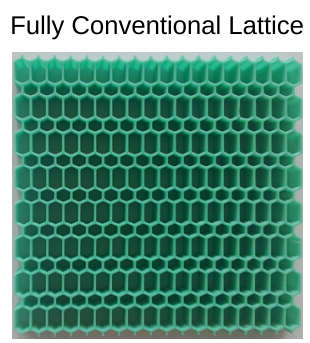

$50 \%$

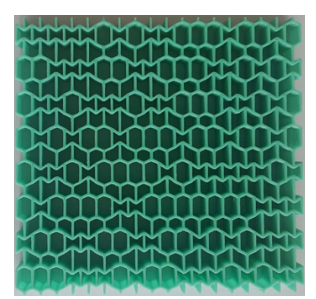

Radial from A-UC to C-UC

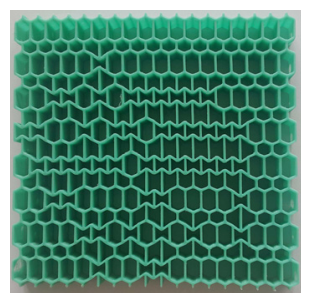

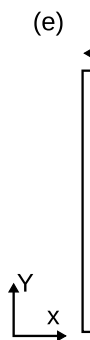

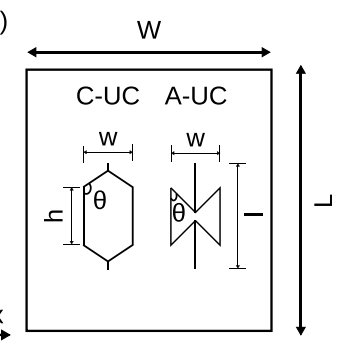

$75 \%$

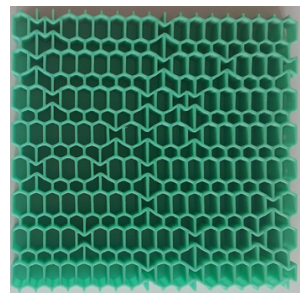

Radial from C-UC to A-UC

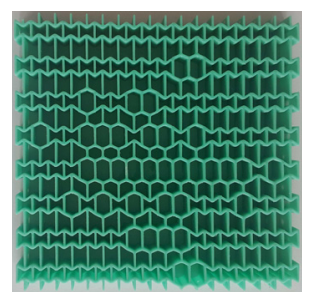

FIG. 1. Elastomer lattice structure with (a) regular and (b and c) random structures. (d) Comparison of experimental results and numerical simulations. The mean \pm standard deviation is shown for the numerical simulations. (e) Geometrical parameters of the unit cells. The total length and width of both unit cells are kept constant. By changing the interior angle $(\theta)$ of unit cells, different geometries can be constructed. $\theta$ can vary between $55^{\circ}$ and $130^{\circ}$. Additional parameters are defined in Table S1 (supplementary material). (d)

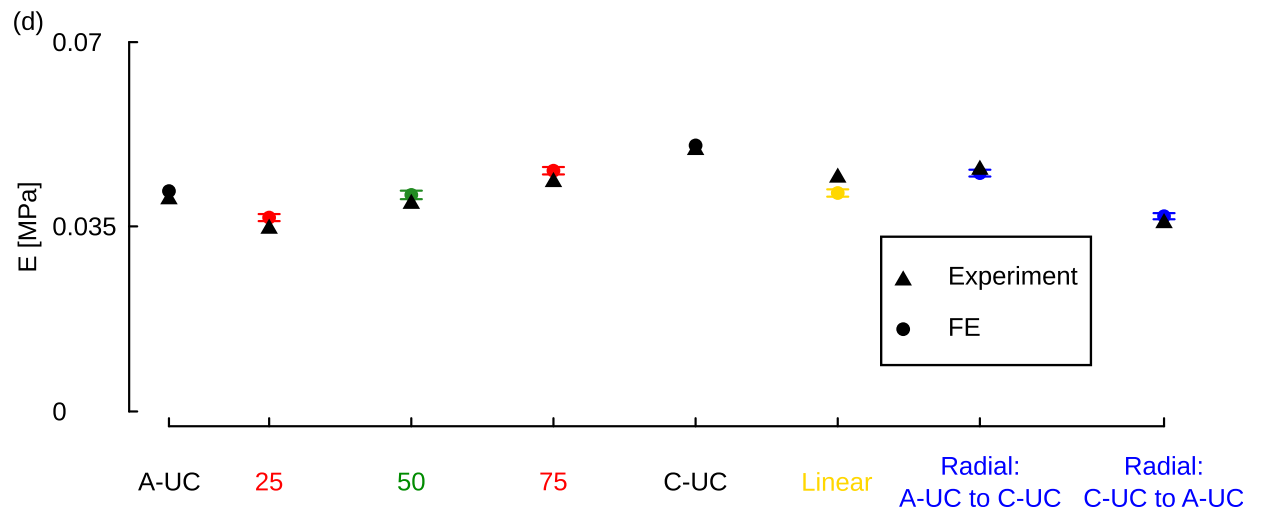


probability of a conventional unit cell in a fully auxetic lattice structure. For instance, in the case of a lattice with the randomness level of $25 \%$, for each unit cell, a random number was created in the range of -0.75 and 0.25 . For every random number greater than zero, a conventional unit cell was positioned in the lattice structure, while the random number below zero resulted in an auxetic unit cell being added to the lattice structure (Fig. 1(b)).

In the case of patterned randomness, conventional unit cells were replaced in a fully auxetic lattice structure by either linear or radial distributions. In the linearly patterned random structures, the probability of having A-UC on the left side of the structure was higher than C-UC. This probability decreased linearly and made to have more C-UC at the very right end section of the structure (Fig. 1(c)). A similar concept was used to introduce a radial random pattern into the structure. In the case of radial randomness from A-UC to $\mathrm{C}$-UC, the probability of having A-UC in the center of the structure was maximum. This probability decreases radially and reaches the minimum likelihood of having A-UC at the boundaries of the lattice structure (Fig. 1(c)).

One thousand simulations were performed for each type of introduced randomness (listed in Table S2 in the supplementary material). A constant level of displacement equal to $U_{x}=2.5 \mathrm{~mm}$ was applied to the FE models. The total reaction force $\left(F_{x}\right)$ was then used to calculate the elastic modulus of the structure. The lateral expansion/contraction was measured for each row of the lattice structure $\left(U_{y, j}\right)$, and the average value of the lateral displacements measured for different rows $\left(U_{y}\right)$ was used to calculate Poisson's ratio of the structure. The nominal stress $(\sigma)$ was defined as the ratio of the applied force to the initial area $\left(A_{0}=L . t_{\text {lattice }}\right)$. Axial strain $\left(\varepsilon_{x}\right)$ and local lateral strains $\left(\varepsilon_{y}\right)$ were determined by $\varepsilon_{x}$ $=U_{x} / W$ and $\varepsilon_{y}=\frac{\sum_{j=1}^{n} U_{y, j}}{n L}$, respectively, in which $n$ is the number of unit cells. Poisson's ratio $(\nu)$ was calculated as $\nu=-\varepsilon_{y} / \varepsilon_{x}$.

Eight samples were fabricated to validate the numerical simulations (Fig. 1). The details of sample preparation and mechanical testing procedures including the specifics of the indirect additive manufacturing technique are presented in the supplementary material. The specimens were tested under quasi-statically applied monotonically increasing tensile load using an INSTRON e10000 dynamic test machine. The experiments were displacement-controlled and were performed at a rate of $4 \mathrm{~mm} / \mathrm{min}$.

The nominal stress $(\sigma)$ was defined as the ratio of the applied force to the initial area $\left(A_{0}\right)$. The axial strain $(\varepsilon)$ was defined as the proportion of the displacement to the free length between two connectors $(W)$. The elastic modulus $(E)$ was calculated from the slope of a linear fit to the stressstrain curve until $5 \%$ strain.

A comparison of FE and experimental results showed less than 6\% difference (Fig. 1(d) and Table S3 in the supplementary material). Those differences may have been caused by the micro-imperfections that arise from the bubbles formed in the ligaments of the lattice structures during the sample preparation process.

The effects of topological design on the elastic modulus-Poisson's ratio duos were then explored using the computational models. First, the effects of the interior angle of each unit cell on the mechanical properties were studied by initially assuming that the angle of A-UC and C-UC is dependent and supplementary. Randomness was then introduced into the models in the ways described before, and the calculated duos of the mechanical properties were compared with those obtained for a lattice with uniform unit cells (solid circles in Fig. 2). Randomness changed Poisson's ratio in the lattice structure, while the elastic modulus remained unchanged (Figs. 2(a) and 2(b), left). In the next step, we (a)

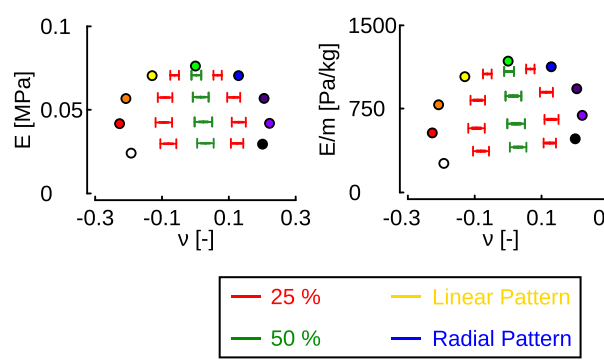

(c)

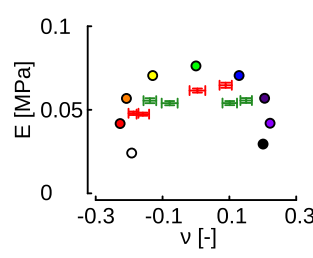

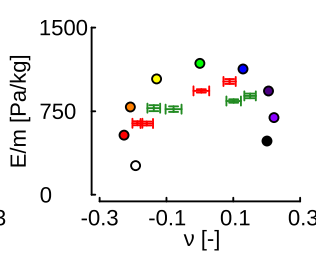

(b)

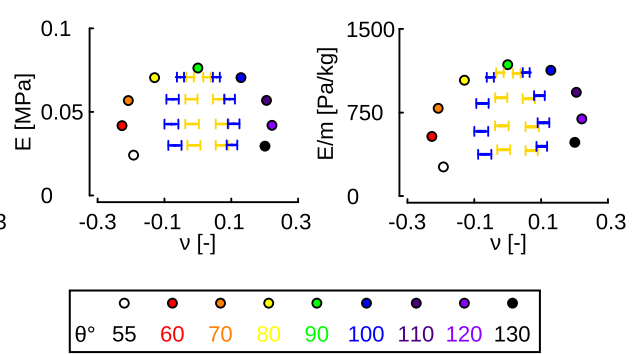

(d)

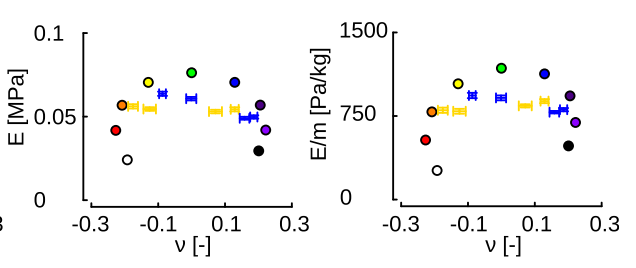

FIG. 2. Comparison of the elastic modulus and Poisson's ratio planes obtained from FE simulations for lattice structures with regular (solid circles) and random microstructures. By the variation of angle $\theta$, various geometries for the unit cells could be achieved. Different microstructural random generation was applied. (a) and (c) Fixed level of randomness equal to $25 \%$ and $50 \%$. (c) and (d) Inducing randomness by a linear and radial pattern. In each part of this figure (a)-(d), the left graph shows the elastic modulus-Poisson's ratio plane, while the right graph shows the specific elastic modulus-Poisson's ratio plane. A-UC and C-UC in lattice structures of (a) and (b) have supplement angles. The unit cells with the highest elastic moduli were combined with unit cells with the highest values of Poisson's ratio (their angles were not necessarily supplementary) in the top row of the figure (a) and (b) to give the graphs in the second row of the figure (c) and (d). 


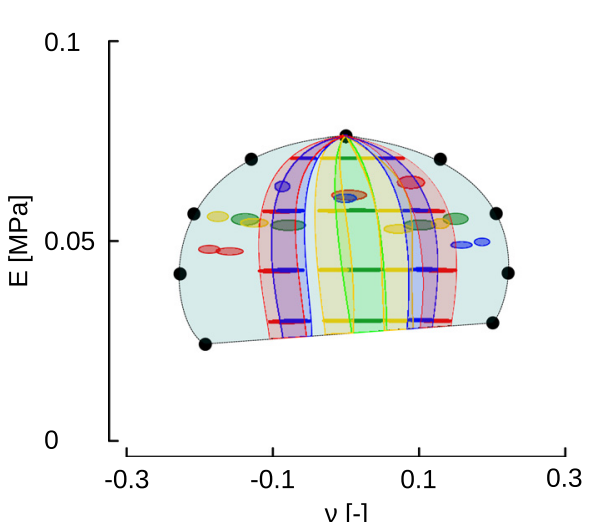

$v[-]$

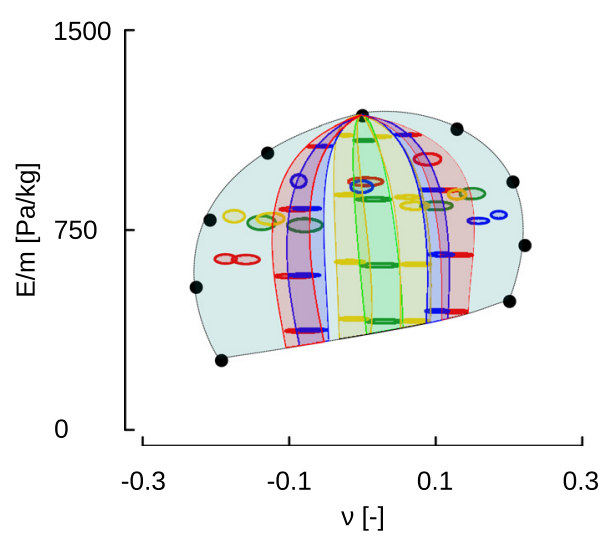

0.3
FIG. 3. Comparison of elastic modulus (left) and specific elastic modulus (right) with Poisson's ratio obtained from FE simulations for regular (solid circles) and random lattice structures. An envelope is drawn on data for the lattice structure with regular unit cells. The results of different methods for the generation of random microstructures are spread as in this envelope. changed the topology of the unit cells independently from each other, i.e., the angles could be independent and not necessarily supplementary (Figs. 2(c) and 2(d), left). The unit cells with the highest elastic moduli were combined with unit cells with the highest values of Poisson's ratio. As an example, auxetic unit cells with $60^{\circ}$ angles (highest Poisson's ratio) were combined with the conventional unit cells with $90^{\circ}$ angles (with the highest elastic modulus). Such random combinations of the unit cells resulted in a broader area of the elastic modulus-Poisson's ratio plane being covered by the elastic properties of the proposed designs (Figs. 2(c) and 2(d), left) as compared to the case where the angles of A-UC and C-UC were supplementary (Figs. 2(a) and 2(b), left).

The normalized [normalized with respect to the elastic stiffness of the solid constituent $\left(E_{s}\right)$ ] elastic modulus of fully auxetic and fully conventional metamaterials varies between 0.033 and 0.105 in our study. This is within the range (0.02-0.1) reported in Ref. 19 for conventional and hybrid metamaterials with comparable ligament thicknesses.

Since changing the topological design and introducing randomness in the structure could influence the relative density of the metamaterials, we normalized the predicted elastic moduli with respect to the mass of the cellular structure, thereby calculating the specific elastic modulus $(E / m)$. The general trends remained the same after normalization of the elastic moduli with some relatively minor changes in the shape of the covered area (Figs. 2(a)-2(d), right). With or without normalization, the fully auxetic and conventional cellular structures created the boundaries of the covered area, while the values inside the area could be obtained by introducing patterned and uniform randomness into the topological design of the metamaterials (Fig. 3).

The ability to independently tailor the elastic modulus and Poisson's ratio and cover a broad area of the associated plane of the elastic properties (Fig. 3) has many practical applications such as in the design process of biomedical implants and scaffolds. Furthermore, this possibility could be used in the design of structurally applied auxetic metamaterials whose functionality is driven by their (low) Poisson's ratio but are simultaneously required to exhibit high stiffness values to ensure sufficient load bearing capacity. It could also serve as a general platform for the rational design of (soft) mechanical metamaterials. From the mechanistic viewpoint, patterned randomness and the interplay between the unit cells with positive and negative Poisson's ratios may be interpreted in analogy with the roles played by dislocations and precipitates in polycrystalline metallic materials. Random positioning of different types of the unit cell has the tendency to change stress flow, thereby resulting in the blockage or faster propagation of the far-field deformations. Those modifications in the deformation paths may be manifested at larger scales as increased or decreased stiffness while not necessarily influencing the lateral deformation and, thus, Poisson's ratio. Additional patterns of randomness and more diverse topological designs of the unit cells are expected to broaden the range of accessible properties even further.

In summary, we proposed patterned randomness in the positioning of auxetic and conventional unit cells as a strategy for independently tailoring the elastic modulus and Poisson's ratio of soft mechanical metamaterials made by indirect additive manufacturing. While metamaterials with a homogenous and uniform arrangement of unit cells create the boundaries of the area covered in the elastic modulusPoisson's ratio plane, the designs incorporating patterned randomness fill the enclosed area. Patterned randomness could, therefore, serve as an additional tool in the rational design of mechanical metamaterials.

See supplementary material for the geometrical parameters of the lattice structures and various randomness patterns.

${ }^{1}$ J. N. Grima and R. Caruana-Gauci, "Mechanical metamaterials: Materials that push back," Nat. Mater. 11(7), 565-566 (2012).

${ }^{2}$ H. M. Kolken and A. Zadpoor, "Auxetic mechanical metamaterials," RSC Adv. 7(9), 5111-5129 (2017).

${ }^{3}$ Z. G. Nicolaou and A. E. Motter, "Mechanical metamaterials with negative compressibility transitions," Nat. Mater. 11(7), 608-613 (2012).

${ }^{4}$ A. A. Zadpoor, "Mechanical meta-materials," Mater. Horiz. 3(5), 371-381 (2016).

${ }^{5}$ R. Lakes, "Negative Poisson's ratio materials: Response," Science 238, 551 (1987).

${ }^{6}$ X. Zheng, H. Lee, T. H. Weisgraber, M. Shusteff, J. DeOtte, E. B. Duoss, J. D. Kuntz, M. M. Biener, Q. Ge, J. A. Jackson et al., "Ultralight, ultrastiff mechanical metamaterials," Science 344(6190), 1373-1377 (2014).

${ }^{7}$ M. Kadic, T. Bückmann, N. Stenger, M. Thiel, and M. Wegener, "On the practicability of pentamode mechanical metamaterials," Appl. Phys. Lett. 100(19), 191901 (2012). 
${ }^{8}$ R. Hedayati, A. Leeflang, and A. Zadpoor, "Additively manufactured metallic pentamode meta-materials," Appl. Phys. Lett. 110(9), 091905 (2017).

${ }^{9}$ S. Janbaz, H. Weinans, and A. A. Zadpoor, "Geometry-based control of instability patterns in cellular soft matter," RSC Adv. 6(24), 20431-20436 (2016).

${ }^{10}$ J. T. Overvelde and K. Bertoldi, "Relating pore shape to the non-linear response of periodic elastomeric structures," J. Mech. Phys. Solids 64, 351-366 (2014).

${ }^{11}$ J. T. B. Overvelde, S. Shan, and K. Bertoldi, "Compaction through buckling in 2D periodic, soft and porous structures: Effect of pore shape," Adv. Mater. 24(17), 2337-2342 (2012).

${ }^{12}$ A. Alderson, K. L. Alderson, D. Attard, K. E. Evans, R. Gatt, J. N. Grima, W. Miller, N. Ravirala, C. Smith, and K. Zied, "Elastic constants of 3-, 4-and 6-connected chiral and anti-chiral honeycombs subject to uniaxial in-plane loading," Composites Sci. Technol. 70(7), 1042-1048 (2010).

${ }^{13}$ R. Gatt, D. Attard, P.-S. Farrugia, K. M. Azzopardi, L. Mizzi, J.-P. Brincat, and J. N. Grima, "A realistic generic model for anti-tetrachiral systems," Phys. Status Solidi B 250(10), 2012-2019 (2013).
${ }^{14}$ H. Wan, H. Ohtaki, S. Kotosaka, and G. Hu, "A study of negative Poisson's ratios in auxetic honeycombs based on a large deflection model," Eur. J. Mech.-A/Solids 23(1), 95-106 (2004).

${ }^{15}$ J. Whitty, F. Nazare, and A. Alderson, "Modelling the effects of density variations on the In-plane Poisson's ratios and Young's moduli of periodic conventional and re-entrant honeycombs-Part 1: Rib thickness variations," Cell. Polym. 21(2), 69-98 (2002).

${ }^{16}$ D. Yang, S. Lee, and F. Huang, "Geometric effects on micropolar elastic honeycomb structure with negative Poisson's ratio using the finite element method," Finite Elements Anal. Des. 39(3), 187-205 (2003).

${ }^{17}$ A. Alderson, J. Rasburn, S. Ameer-Beg, P. G. Mullarkey, W. Perrie, and K. E. Evans, "An auxetic filter: A tuneable filter displaying enhanced size selectivity or defouling properties," Ind. Eng. Chem. Res. 39(3), 654-665 (2000).

${ }^{18}$ K. Wang, Y.-H. Chang, Y. Chen, C. Zhang, and B. Wang, "Designable dual-material auxetic metamaterials using three-dimensional printing," Mater. Des. 67, 159-164 (2015).

${ }^{19}$ K. R. Olympio and F. Gandhi, "Zero Poisson's ratio cellular honeycombs for flex skins undergoing one-dimensional morphing," J. Intell. Mater. Syst. Struct. 21(17), 1737-1753 (2010). 